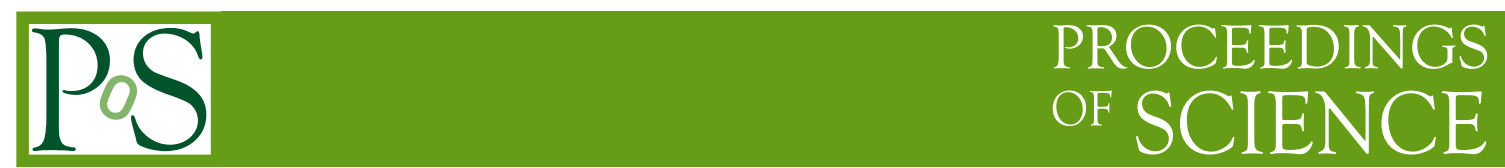

\title{
Current status of gravitational wave observations
}

\author{
Nigel T. Bishop* \\ Rhodes University \\ E-mail: n.bishop@ru.ac.za
}

We review LIGO and VIRGO detection events reported so far, as well as future prospects. We also review the prospects for gravitational wave measurements, but in lower frequency bands, from pulsar timing arrays, and from the space system LISA.

5th Annual Conference on High Energy Astrophysics in Southern Africa

4-6 October, 2017

University of the Witwatersrand (Wits), South Africa

${ }^{*}$ Speaker. 


\section{Introduction}

Advanced LIGO has completed its first observation runs (O1: 2015.09.29 to 2016.01.12, O2: 2016.11.30 to 2017.08.25); Virgo has also come on line and completed its first observation run 2017.07.28 to 2017.08.25. To date, there have been reported 5 definite and 1 probable black hole mergers, GW150914, GW151226, GW170104, GW170608, GW170814 and LVT151012; and one neutron star merger event, with an electromagnetic counterpart, GW170817. Other signals that were possibly expected, have not been observed, including burst events such as Galactic supernovas and cosmic string interactions, and continuous sources caused by the rotation of a nonaxisymmetric body examples being the Crab, Vela and other pulsars. Even such non-detections provide constraints on astrophysical models.

The limited results obtained so far have already had an impact on theoretical astrophysics, since an expalanation for the origin of black holes with mass $\mathscr{O}\left(30 M_{\odot}\right)$ is required. As will be discussed later, an enormous increase in detections is expected over the next few years. Thus gravitational wave $(\mathrm{GW})$ observations will provide strong constraints on the modeling of various astrophysical and physical processes.

This review starts with an introduction to the basic theory of GW generation and propagation; then summarizes the functioning of a LIGO machine; the events that have been detected to date are described, as well as the implications for physics and astrophysics. We then look at future prospects, in terms of the current LIGO facilities as well as planned ones; and other plans for GW detection, i.e. the satellite system LISA and pulsar timing arrays.

\section{GW basics}

The simplest approach to GWs is to linearize Einstein's equations, usually about a flat Minkowski background, and it is found that, to leading order, the GW field takes the form

$$
d s^{2}=-d t^{2}+d x^{2}+\left(1+h_{+}(t, x)\right) d y^{2}+\left(1-h_{+}(t, x)\right) d z^{2}+2 h_{\times}(t, x) d y d z,
$$

using Cartesian coordinates $(t, x, y, z)$ for a plane $\mathrm{GW}$ propagating in the $x$-direction. The functions $h_{+}(t, x), h_{\times}(t, x)$ are independent and each satisfies the wave equation $\left(\partial_{t}^{2}-\partial_{x}^{2}=0\right)$. They constitute the two polarization modes of the GW; if the $(x, y)$ axes are rotated through $\pi / 4$ about the $z$-axis, then the two modes are interchanged. A GW detector comprises two perpendicular arms each of length $L$, and laser light travels along each arm and back again. The difference in travel time between the two arms is measured as an interference fringe; see Fig. 1. For a more detailed discussion of the operation of a LIGO detector, see for example [1]. If, for a given wave, the arms lie along the $y$ and $z$ axes, then $2 L h_{+}$is measured; whereas if the arms are in the $y, z$ plane but at $\pi / 4$ to the axes, then $2 L h_{\times}$is meaured. If one of the arms is in the direction of propagation of the wave, then its length will not change; but the other arm will be transverse to the wave, and the relative change of length will be half that of the case where both arms are transverse to the wave. The one case in which no effect will be recorded is if the wave is linearly polarized, which means that either $h_{+}$or $h_{\times}$is zero, and the arms are oriented such that the zero component is measured. The current LIGO detectors have arms of length $4 \mathrm{~km}$, and for VIRGO the length is $3 \mathrm{~km}$. This 


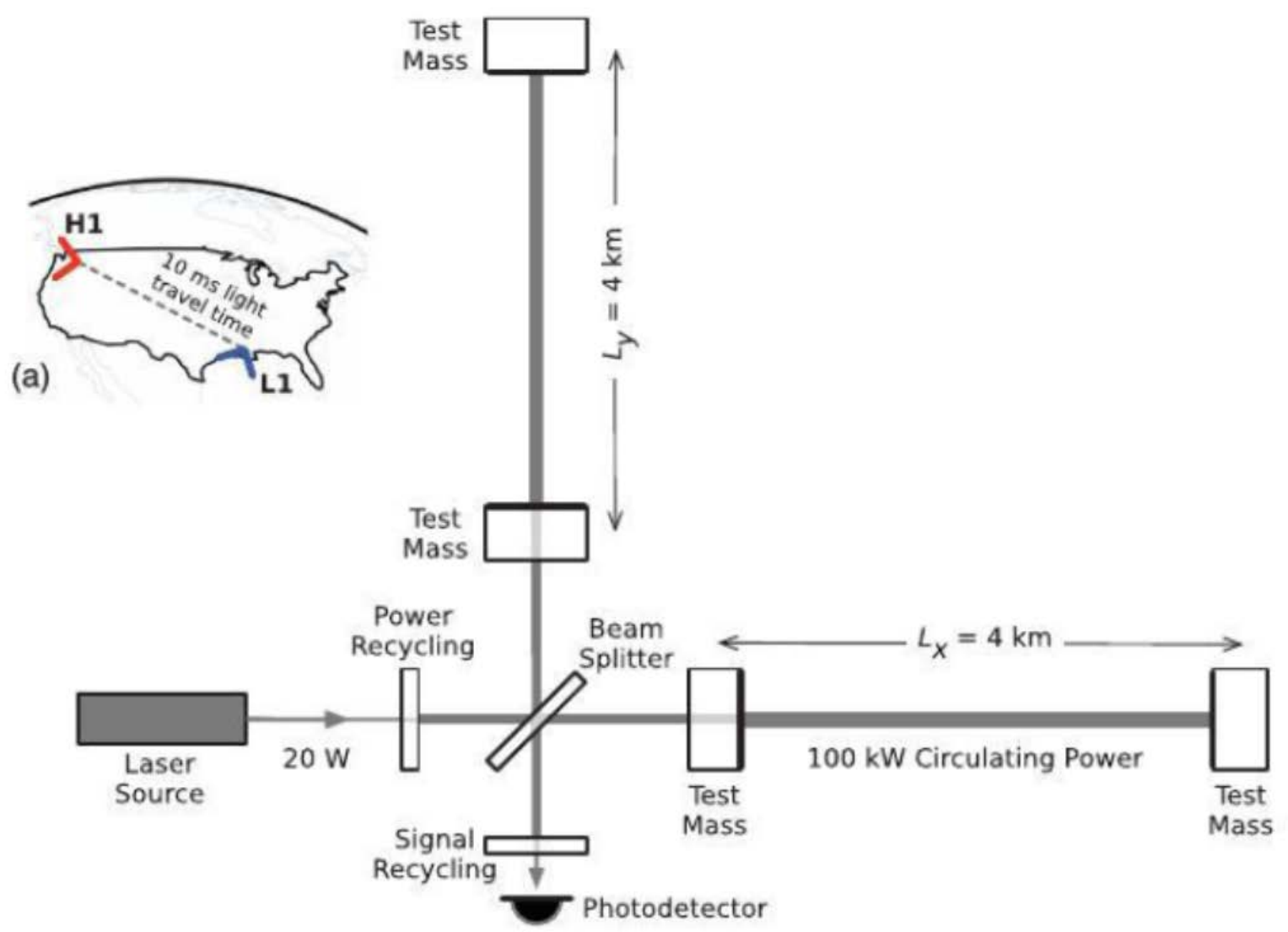

Figure 1: Illustration of the schematics of the LIGO facilities, adapted from [2]

leads to the detectors being sensitive only to signals in the frequency range approximately $20 \mathrm{~Hz}$ to $2000 \mathrm{~Hz}$.

The generation of GWs by a source is often described by Einstein's quadrupole formula [3, 4], which is valid for systems where the relative velocities are small. For a binary (see Fig. 2) comprising two masses, $M_{1}$ and $M_{2}$ in circular orbit of diameter $r_{0}$ and with angular velocity $\omega$, the formula gives [5]

$$
\left(h_{+}, h_{\times}\right)=-\frac{8 M_{1} M_{2} r_{0}^{2} \omega^{2}}{\left(M_{1}+M_{2}\right) r} \times\left(\left(1+\cos ^{2} \theta\right) \cos (2 \omega t-2 \phi), 2 \cos \theta \sin (2 \omega t-2 \phi)\right),
$$

indicating that the wave frequency is twice that of the orbital frequency. The wave magnitude falls off as $1 / r$. Thus, if detection sensitivity increases by a factor 2 , a source could be 2 times further, so the volume searched and consequently the expected event detection rate would be $2^{3}=8$ times larger.

The energy carried away by GWs is

$$
\frac{d E}{d t}=-\frac{1}{4 \pi} \oint_{S}\left(\partial_{t} h_{+}\right)^{2}+\left(\partial_{t} h_{\times}\right)^{2}
$$




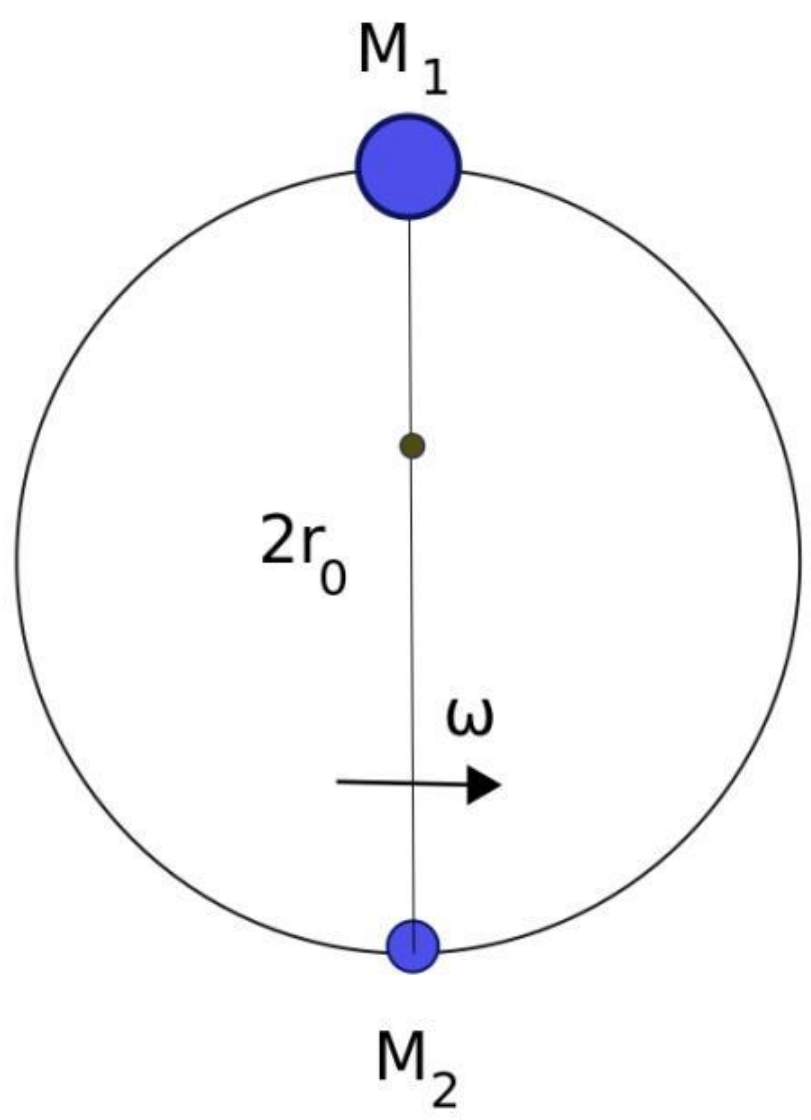

Figure 2: Two masses, $M_{1}$ and $M_{2}$ in circular orbit diameter $2 r_{0}$ rotating at angular velocity $\omega$.

where $S$ is a spherical shell at large, constant $r$. (Actually, the energy emitted has historically been a controversial issue, which was resolved only in 1962 by Bondi et al. [6].) The rate of energy emission of GWs is equated to the rate of loss of Newtonian orbital energy, yielding

$$
\frac{d E}{d t}=-\frac{M_{1}^{2} M_{2}^{2}\left(M_{1}+M_{2}\right)}{5 r_{0}^{5}}=\frac{d}{d t}\left(-\frac{M_{1} M_{2}}{4 r_{0}}\right)=\partial_{t} r_{0} \frac{M_{1} M_{2}}{4 r_{0}^{2}} .
$$

Applying the relation between $\omega$ and $r_{0}$ using Newtonian orbit theory (Kepler's law), we find

$$
\omega=\sqrt{\frac{M_{1}+M_{2}}{8 r_{0}^{3}}}, \frac{\partial_{t} \omega}{\omega}=-\frac{3}{2} \frac{\partial_{t} r_{0}}{r_{0}},
$$

so that $r_{0} \propto \omega^{-2 / 3}$. We can now use Eq. (2.5) in Eq. (2.4) to replace $\partial_{t} r_{0}$ and $r_{0}$ by $\partial_{t} \omega$ and $\omega$ to obtain the chirp mass formula

$$
\mathscr{M} \equiv \frac{\left(M_{1} M_{2}\right)^{3 / 5}}{\left(M_{1}+M_{2}\right)^{1 / 5}}=\left(\frac{5}{96} \pi^{-8 / 3} f^{-11 / 3}\left(\partial_{t} f\right)\right)^{3 / 5},
$$

where $f$ is the wave frequency, related to the orbital angular velocity by $\omega=\pi f$. The key point is that both $f$ and $\partial_{t} f$ are determinable entirely from LIGO data, and thus so is the chirp mass. 
Observed changes to the orbital frequency of the Hulse-Taylor binary pulsar, and other systems, have provided confirmation of this formula since the 1970s.

We use Kepler's law Eq. (2.5) to replace $r_{0}$ by $f$ in Eq. (2.2), and find the wave amplitude

$$
\left(A_{+}, A_{\times}\right)=\frac{2 \mathscr{M}^{5 / 3}(\pi f)^{2 / 3}}{r}\left(\left(1+\cos ^{2} \theta\right), 2 \cos \theta\right)
$$

where $\left(h_{+}, h_{\times}\right)=\left(A_{+} \cos (2 \omega t-2 \phi), A_{\times} \sin (2 \omega t-2 \phi)\right)$. Thus, once the chirp mass is determined and if the polarization modes can be separated so that the inclination $\theta$ of the source as seen by the detector is constrained, then the above result may be used to constrain the distance to the source.

The redshift $z$ of the source causes frequencies in the source frame to be higher than in the detector frame by a factor $(1+z)$, and this gives a correction that source frame masses are smaller than detector frame masses by a factor $1 /(1+z)$. In the absence of an optical counterpart and direct measurement, the redshift is estimated from the distance using Hubble's law.

Qualitatively, the system evolves with the orbital diameter slowly decreasing, and the wave magnitude, wave frequency and orbital velocity slowly increasing. Once the orbital velocity (i.e. $\left.r_{0} \omega\right)$ is not negligible compared to the velocity of light, then alternative methods need to be used for accurate waveform estimation; this is essential, since the wave amplitude is largest, and therefore more detectable, in this regime. These methods may be analytic (post-Newtonian, postMinkowskian and self-force), or they may require numerical evolution of the Einstein equations; but they are not discussed further in this review. However, the quadrupole formula gives results that are accurate as order of magnitude estimates even outside its domain of applicability. Except, possibly, in the case of two neutron stars, the end product will be a black hole, and black hole perturbation theory [7] can be used to estimate the post-merger waveform. This gives

$$
\left(h_{+}, h_{\times}\right) \sim \exp \left(-\frac{t}{\tau}\right) \cos \left(\frac{\omega t}{2 \pi}\right), \text { where } \tau=0.056 \frac{M}{M_{\odot}} \mathrm{ms}, \quad \omega=12000 \frac{M_{\odot}}{M} \mathrm{~Hz},
$$

and where $M$ is the total mass of the system.

The amplitude and frequency increase until they reach maxima at merger. The maximum frequency is of order

$$
f_{\max } \approx \frac{M_{\odot}}{\mu} 5000 \mathrm{~Hz}
$$

where $\mu=M_{1} M_{2} /\left(M_{1}+M_{2}\right)$ is the reduced mass of the system Thus LIGO would not see events with $M_{1}, M_{2} \gtrsim 500 M_{\odot}$ since throughout the inspiral and merger the wave frequency would be too low. Let $f_{\text {in }}$ be the frequency when the signal is first "in band"; normally, $f_{\text {in }} \approx 20 \mathrm{~Hz}$, but if the signal is weak it could be larger. Integrating the chirp formula Eq. (2.6), we find that the signal is viewed in the detector for a time

$$
t=\frac{5}{256 \pi^{8 / 3} \mathscr{M}^{5 / 3} f_{\text {in }}^{8 / 3}},
$$

where the integration is taken until $f \rightarrow \infty$ representing merger. For example, GW150914 has $\mathscr{M} \approx 30 M_{\odot}$ and $f_{\text {in }} \approx 50 \mathrm{~Hz}$; this leads to $t=0.06 \mathrm{~s}$. On the other hand, a system comprising 2 neutron stars each of mass $1.5 M_{\odot}$ has $\mathscr{M} \approx 1.3 M_{\odot}$; if the signal is detectable at $20 \mathrm{~Hz}$, then it would be in-band for 138s, so in such a case it may be possible to alert other observatories about an impending merger before it happens. 
From Eq. (2.4) we have

$$
\partial_{t} r_{0}=-\frac{4 M_{1} M_{2}\left(M_{1}+M_{2}\right)}{5 r_{0}^{3}}
$$

which may easily be integrated to derive the time to coalescence

$$
t_{c}=\frac{5 r_{0}^{4}}{16 M_{1} M_{2}\left(M_{1}+M_{2}\right)} .
$$

For example, two $10 M_{\odot}$ black holes 10 million $\mathrm{km}$ apart, will take 0.9 billion years until merger; and approximately the same time would be taken by two $50 M_{\odot}$ black holes 25 million km apart. A larger separation would not lead to merger in less than a Hubble time. Thus in order to understand why black hole mergers are observed, we need to understand the astrophysical processes that drive the black holes close enough for GW damping to be effective at driving the inspiral.

The angle of incidence of a GW onto the plane of a detector is completely unknown if there is only one detector, is constrained to lie on a cone if there are two detectors, and is well-constrained if there are 3 or more detectors (which is why concurrent observations by several detectors are so important). With only one detector, the magnitude of the wave is uncertain by a factor 2 , which translates to uncertainty by a factor of 2 in estimating the distance to the event. Further, the angle $\theta$ between the direction of wave propagation and the orbital axis of the source is unknown, leading to an uncertainty in the magnitude of the wave and thus of the distance to the source. These degeneracies may be broken in a number of ways. The source may be well-localized on the sky if the event is seen in multiple detectors, or if an electromagnetic counterpart is identified. Further the angle $\theta$ may be constrained if the two polarization modes can be disentangled, which means that the signal must be seen in multiple detectors with the arms in different relative orientations. The GW signal is not solely quadrupolar, but involves higher order harmonics at higher time-frequencies. If the GW signal is at large enough SNR (Signal to Noise Ratio) for a higher order harmonic to be estimated, then $\theta$ can be constrained since the $\ell=2$ and $\ell=4$ modes have different $\theta$ dependency.

We have considered only the waveform generated by circular orbits, but in general orbits are elliptical with varying degrees of eccentricity. The orbital velocity is highest at periastron, and consequently so also are GW emission and the effective radiation back reaction. The result is that

the eccentricity $e$ decays much faster than the orbital diameter, specifically $e \propto r_{0}^{19 / 12}$ [8]. GWs are detectable only in the final stages of the inspiral, by which stage the orbits are therefore expected to have become circularized. One exception could be if the binary is not isolated but is part of a larger system that injects eccentricity to the binary, but such a scenario is unlikely.

\section{GW events that have been detected}

In order to determine the astrophysical parameters of an event, the starting point is a template bank of merger waveforms over the parameter space to be explored. For black hole mergers, both the phase and the magnitude of the GW scale with the total mass, so the parameter space comprises the mass ratio $q=M_{1} / M_{2}$ and the spins $\boldsymbol{S}_{1}, \boldsymbol{S}_{2}$. In practice, the template bank is constructed using analytic methods, with various parameters in the expansions fixed by fitting the resultant waveforms to the output of numerical relatvity simulations. The mismatch between the calculated waveform 
Table 1: Binary black hole mergers detected as GW events 2015 - 2017.

$\begin{array}{lccccc}\text { GW Event } & 150914 & 151226 & 170104 & 170814 & 170608 \\ \text { Reference } & {[2]} & {[9]} & {[10]} & {[11]} & {[12]} \\ \text { Primary mass } m_{1} / M_{\odot} & 36.2_{-3.8}^{+5.2} & 14.2_{-3.7}^{+8.3} & 31.2_{-6.0}^{+8.4} & 30.5_{-3.0}^{+5.7} & 12_{-2}^{+7} \\ \text { Secondary mass } m_{2} / M_{\odot} & 29.1_{-4.4}^{+3.7} & 7.5_{-2.3}^{+2.3} & 19.4_{-5.9}^{+5.3} & 25.3_{-4.2}^{+2.8} & 7_{-2}^{+2} \\ \text { Total mass } M / M_{\odot} & 65.3_{-3.4}^{+4.1} & 21.8_{-1.7}^{+5.9} & 50.7_{-5.0}^{+5.9} & 55.9_{-2.7}^{+3.4} & 19_{-1}^{+5} \\ \text { Final mass } M_{f} / M_{\odot} & 62.3_{-3.1}^{+3.7} & 20.8_{-1.7}^{+6.1} & 48.7_{-4.6}^{+5.7} & 53.2_{-2.5}^{+3.2} & 18_{-0.9}^{+4.8} \\ \text { Final spin } a_{f} & 0.68_{-0.06}^{+0.05} & 0.74_{-0.06}^{+0.06} & 0.64_{-0.20}^{+0.09} & 0.70_{-0.05}^{+0.07} & 0.69_{-0.05}^{+0.04} \\ \text { Radiated energy } E / M_{\odot} c^{2} & 3.0_{-0.4}^{+0.5} & 1.0_{-0.2}^{+0.1} & 2.0_{-0.7}^{+0.6} & 2.7_{-0.3}^{+0.4} & 0.85_{-0.17}^{+0.07} \\ \text { Luminosity distance }(\mathrm{Mpc}) & 420_{-180}^{+150} & 440_{-190}^{+180} & 880_{-390}^{+450} & 540_{-210}^{+130} & 340_{-140}^{+140} \\ \text { Sky localization }\left(\mathrm{deg}^{2}\right) & 230 & 850 & 1200 & 60 & 860\end{array}$

$\left(h_{c}\right)$ and the observed waveform $\left(h_{o}\right)$ is defined as

$$
\mathscr{M}\left(h_{c}\right)=1-\left\langle h_{c}, h_{o}\right\rangle
$$

where the inner product is normally evaluated in the Fourier domain; includes the detector response function; and satisfies $0 \leq\left\langle h_{1}, h_{2}\right\rangle \leq 1$, being $=1$ iff $h_{1}=$ constant $\times h_{2}$. Then $\mathscr{M}\left(h_{c}\right)$ is minimized over the template bank.

The properties of the binary black hole merger events detected to date during the $O 1$ and $O 2$ observation runs are summarized in Table 1. 0.4s after GW150914 the Fermi Gamma-ray Burst Monitor observed a weak gamma-ray transient (GBM150914) of duration 1s [13]; but this observation was not confirmed by other detectors. No optical, radio or neutrino counterparts were observed. No counterparts to other BBH GW events were reported. The lack of multi-messenger counterparts is not surprising, because they would require the presence of some form of matter: a system comprising black holes only would not produce any signal other than GWs.

The detection events show clearly the existence of a population of intermediate mass black holes. This is interesting because the end-state of a star had not been expected to lead to a black hole more massive than about $10 M_{\odot}$. Thus, an important question is the formation channel of black holes in the mass range $20 M_{\odot}$ to $40 M_{\odot}$.

The consistency between the observed and predicted black hole merger waveforms amounts to a test of general relativity in the strong field regime. On the other hand, the data do not exclude the possibility of the merging objects being some form of exotic compact object other than black holes, nor does it exclude some alternative theories of gravity.

An exciting result from the $O 2$ observation run was the first detection of a binary neutron star merger GW170817 [14, 15]; see Fig. 3. The Fermi Gamma-ray Burst Monitor observed a burst about $1.7 \mathrm{~s}$ after the merger time in the GW data; this signal was also observed by INTEGRAL. The GW signal was seen in both LIGO detectors, and had a duration of about 100s. The Virgo signal was of low amplitude, and is not discernible in Fig.3; it contributed to constraining the sky position (but not other source parameters) to $31 \mathrm{deg}^{2}$. Searches for an electromagnetic counterpart yielded results about 11 hours after the GW event, identifying a bright optical transient source in the galaxy NGC 4993 at a distance of about 40Mpc. This observation was confirmed by a number 
of observatories around the world, including SALT in South Africa. There followed a worldwide multi-messenger search for additional signals. The details are given in [15] and references therein. In summary, signals were observed in the X-ray (after a delay of 9 days), UV, optical, IR and radio (after a delay of about 16 days, and including MeerKAT) bands. No neutrino flux was recorded, and subsequent searches for $\gamma$-rays by ground based facilities including H.E.S.S. were also unsuccessful.

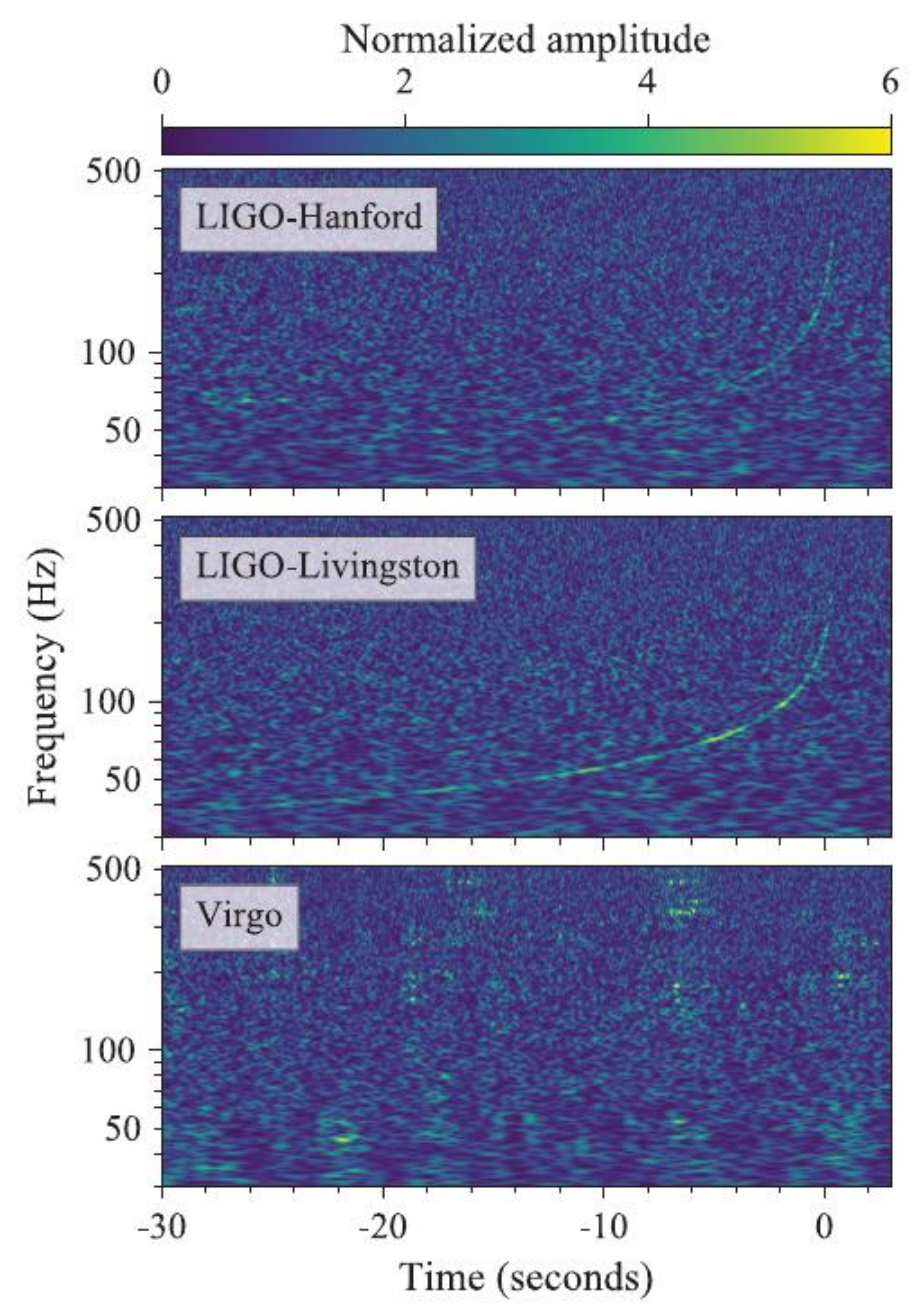

Figure 3: Time-frequency plot of the LIGO-Hanford, LIGO-Livingston and Virgo data for GW170817; from [14].

Taking account of all the observations, there is strong evidence that GW170817 was a neutron star merger followed by a short gamma ray burst (sGRB) and a kilonova with radioactive decay of $r$-process nuclei in the ejecta. The mass of the system, in the form of the chirp mass, is well constrained, $\mathscr{M}=1.188_{-0.002}^{+0.004} M_{\odot}$, and the constraints obtained on the individual and total masses are $1.17 M_{\odot}<M_{1}, M_{2}<1.60 M_{\odot}, M_{1}+M_{2}=2.74_{-0.01}^{+0.04} M_{\odot}$. In classical general relativity, GWs propagate at the speed of light $(c)$. The time difference between the arrival of the GW signal and the GRB lead to a constraint on the difference between the two speeds to be in the range $-3 \times 10^{-15} \mathrm{c}$ 
to $+7 \times 10^{-16} c$. The internal structure of a neutron star has no effect on the GW signal at early times where only the masses are significant, but it does have an effect at later times and at merger. The GW data constrains the tidal deformation parameter $\Lambda$. The results provide an upper limit on $\Lambda$ which is about the same as that obtained previously by other means. The results somewhat favour models that have smaller radii, and thus softer equations of state.

It has been proposed [16] that the Hubble constant can be measured using GW data, since, in principle, GW data alone is sufficient to determine the distance to the source. Here [17], the precise sky localization means that there is no uncertainty in the orientation of the detectors relative to the GW propagation vector. The uncertainty in the orientation of the source with respect to the line of sight to the detector, i.e. $\theta$ in Eq. (2.2), is constrained since the 3 detectors are at different orientations and so provide information about the polarization. It is found that $144 \mathrm{deg}<\theta<180 \mathrm{deg}$, i.e. the source was viewed nearly face-on with the rotation being clockwise. The estimate obtained for the distance to the source is $43.8_{-6.9}^{+2.9} \mathrm{Mpc}$, which is consistent with astronomical estimates of the distance to NGC 4993. The value obtained for the Hubble constant is $70.0_{-8.0}^{+12.0} \mathrm{~km} \mathrm{~s}^{-1} \mathrm{Mpc}^{1}$. The uncertainty in the value should be reduced in future as more events are discovered. At this stage, the value obtained is consistent with estimates from both cosmic microwave background data and supernovae (SN1a), and does not shed light on the tension between these values.

\section{Future prospects}

Currently, there are 3 detectors: 2 in the USA, LIGO Hanford and LIGO Livingston, and Virgo in Italy. The detectors are not operating at design sensitivity, and the goal is to improve the current sensitivity by a factor of 2 . This translates into being able to search for sources in a volume that is $2^{3}=8$ times larger, and so to an expected increase in the event detection rate by a factor of 8 . In addition, detectors are under construction: KAGRA, Japan, with observations scheduled to start in 2020; and INDIGO, India, with observations scheduled to start in 2022. Further systems such as the Einstein Telescope have been discussed, but are still at the planning and fund-raising stages.
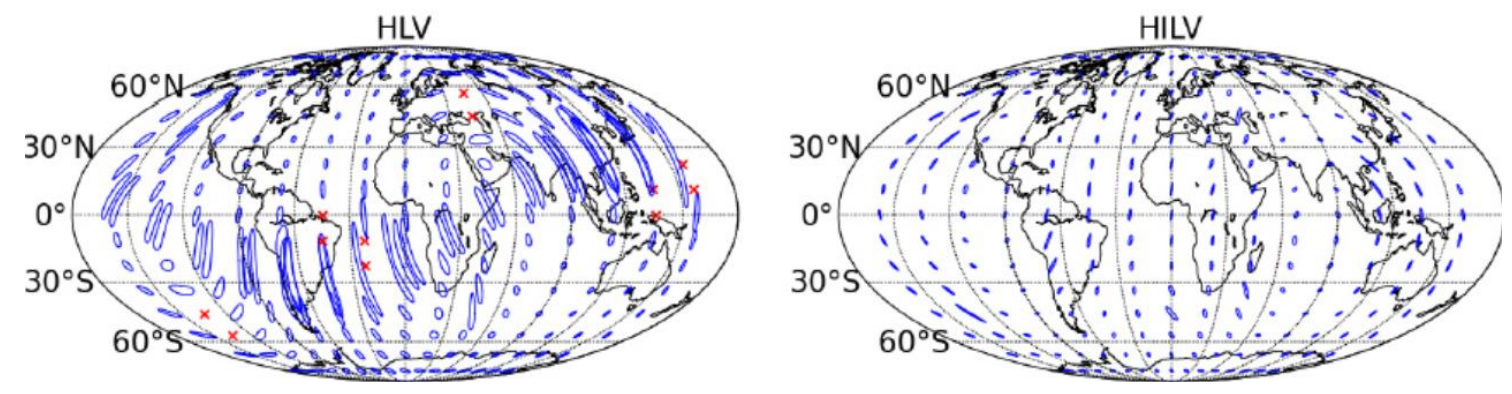

Figure 4: Illustration of expected sky localization with 3 detectors (LIGO-Hanford, LIGO-Livingston and Virgo) in the left panel, and with the addition of a $4^{\text {th }}$ detector, INDIGO, in the right panel. Adapted from [1].

The advantage of multiple detectors is improved sky localization (see Fig. 4), as well being able to measure the polarization modes, with the constraints becoming tighter as the number of detectors observing the GW increases. A further advantage is lock-time: the detectors have scheduled maintenance, and even during observation runs they are in lock, i.e. taking data, only for about 
$75 \%$ of the time. More detectors increases the probability that at a given time at least 2 detectors will be in lock. Taking all these factors into account, the expectation is that by the early 2020 s black hole merger events of order 1 per day will be observed.

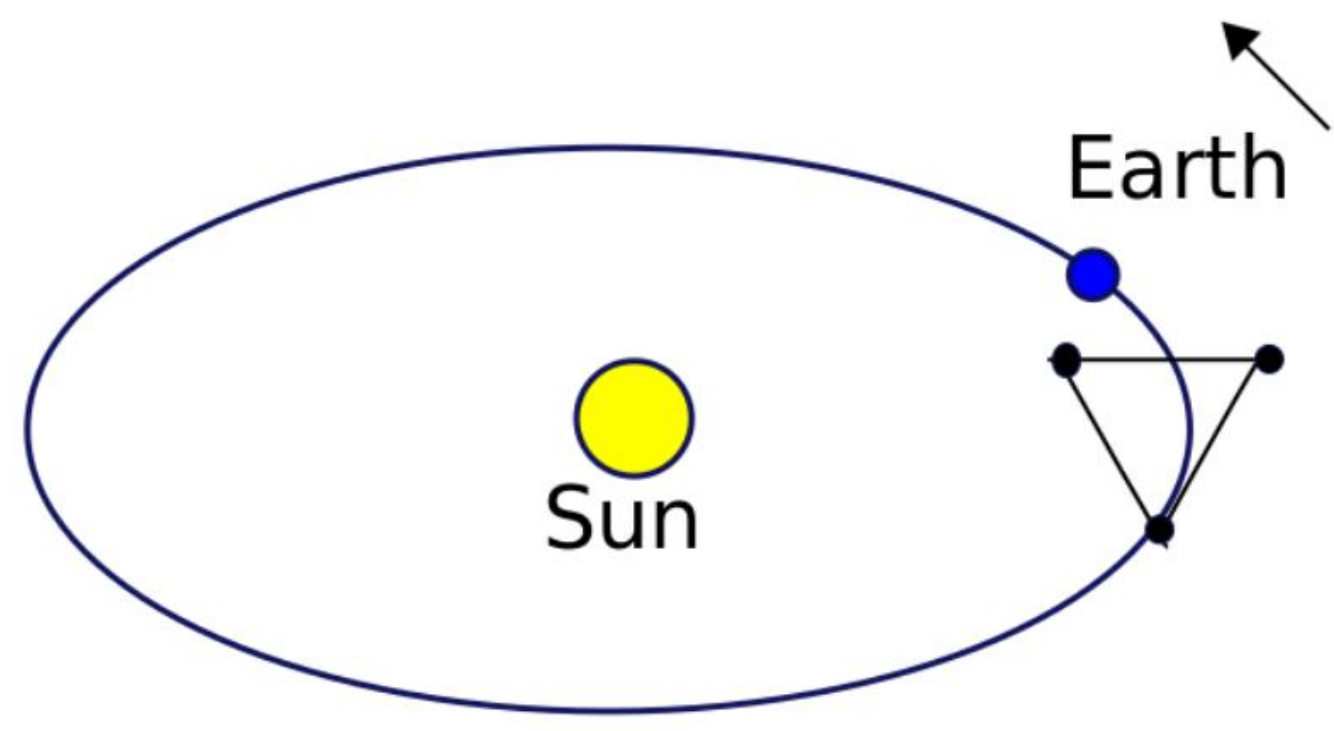

Figure 5: Illustration of the orbits of the LISA satellites following the Earth around the Sun.

LISA pathfinder was launched on 3 December 2015, and tested technology to achieve freelyfalling test masses from 1 March 2016; the mission ended 18 July 2017. The results are 5 times more accurate than target, and meet the LISA design specifications to within a factor of 1.25. Consequently, the European Space Agency project has scheduled LISA for launch in 2034. It will comprise 3 satellites in heliocentric orbit, following the Earth, and forming an equilateral triangle with sides of 2.5 million km; see Fig. 5. The triangle will not be fixed relative to the Earth, but will make a complete rotation once per year. Each satellite will have a laser transmitter, receiver, and interferometer, as well as a reference test mass inside the satellite. The test mass is protected from external influences and will follow, very precisely, a spacetime geodesic. Further details are given in the mission proposal [18]. The design allows the two polarization modes to be measured. Sky localization will not be possible for a burst event, but if GWs from a source are detected over at least several weeks, the change in orientation of the system as it moves around the Sun will allow the source to be localized. The arm length means that LISA will be sensitive to sources in a different frequency band compared to LIGO, about $10^{-1} \mathrm{~Hz}$ to $10^{-4} \mathrm{~Hz}$. Consequently, the merger events relevant to LISA are those involving supermassive black holes, either with another supermassive black hole or with a stellar mass object. There are a number of known binaries in our Galaxy comprising white dwarfs, neutron stars or $\mathscr{O}\left(10 M_{\odot}\right)$ black holes, with orbital periods that produce GWs in the LISA frequency band; these can be used as verification signals for LISA, and doubtless LISA will discover more such systems. $\mathscr{O}\left(10 M_{\odot}\right)$ black hole binaries are visible in the LISA band weeks or months before merger, so permitting a merger event to be predicted so that 
optical telescopes can be pointed towards it when it occurs.

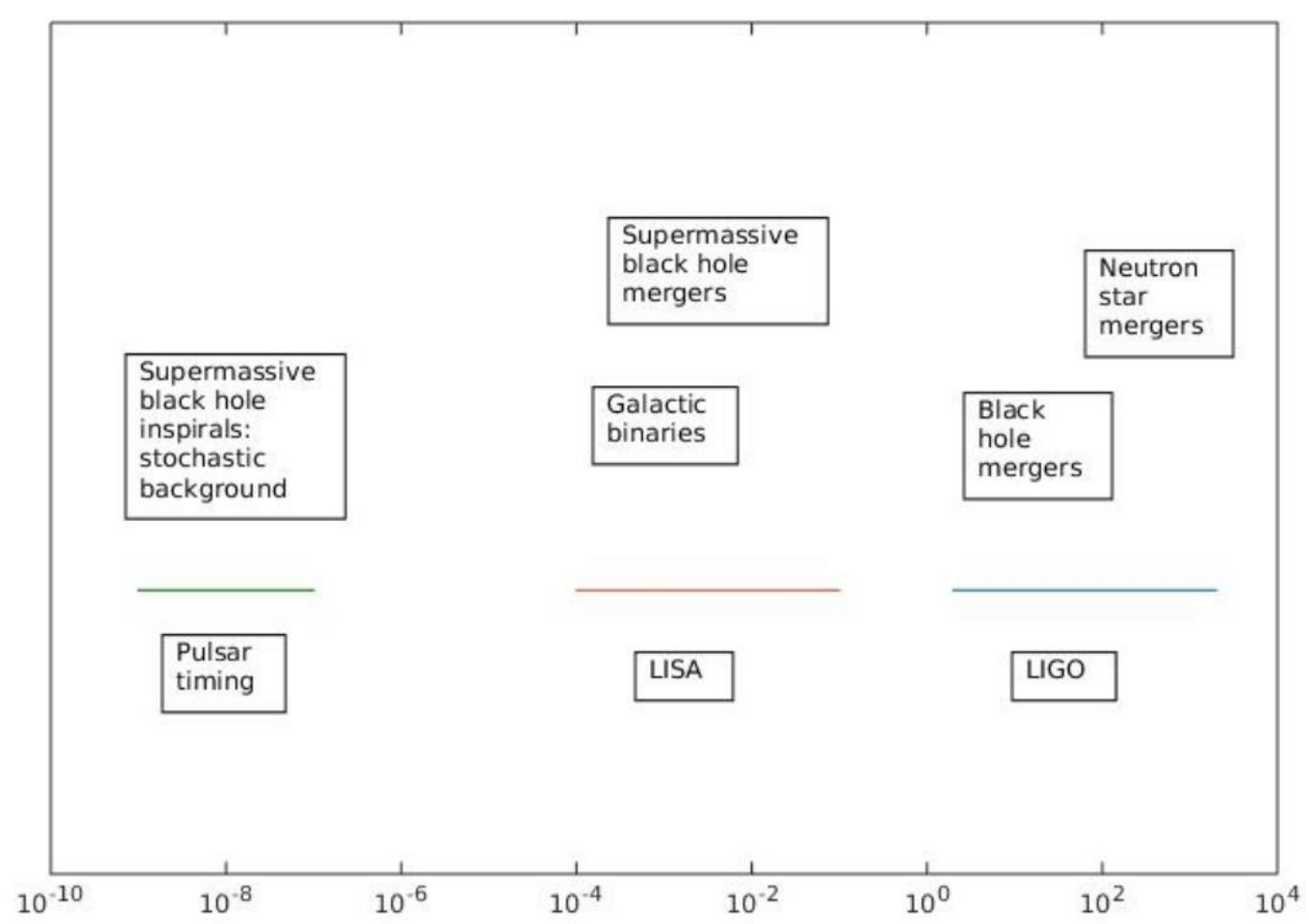

Figure 6: LIGO, LISA and Pulsar Timing frequency ranges and expected astrophysical events for detection

Millisecond pulsars are highly accurate clocks, beating at about $10^{3} \mathrm{~Hz}$. Pulsar timing projects record accurately the time of arrival of each pulse from a particular pulsar, and compare it to the expected time of arrival assuming a uniform time between the pulses. This difference is recorded as the timing residual. (In practice, the process is rather more complicated, because it is only when averaged over a number of pulses that uniform pulse emission occurs, and further there may be glitches in pulse emission; thus the data analysis is statistical rather than direct). If a GW passes the Earth, then the timing residuals will oscillate according to the form of the GW, e.g. for a continuous signal the residuals will oscillate sinusoidally. In addition to searching for GWs from a specific source, pulsar timing can be used to search for a stochastic background. Given data from two pulsars at angle $\theta$ between their directions, then the signals should be correlated according to the Hellings-Downs formula [19]. Pulsar timing is sensitive to GWs at very low frequencies (about $10^{-7} \mathrm{~Hz}$ to $10^{-9} \mathrm{~Hz}$ ), which, for example, are generated by super-massive black hole binaries well before merger. Pulsar timing data collection has been ongoing since 2004, but to date has only been able to set upper limits; probably the detction of an event or of the stochastic background will require several more years. The International Pulsar Timing Array is a collaboration that coordinates the efforts of radio astronomers in a number of countries, with more facilities participating with time. In South Africa, MeerKAT participates in pulsar timing, and it is also one of the science goals of the SKA. Further details on pulsar timing projects may be found, for example, in the 
review [20].

The frequency ranges of the various detection systems, as well as the astrophysical events that are expected to be detected, are summarized in Fig. 6.

\section{Conclusion}

We are now in the age of gravitational wave astronomy. Within a few years, there should be 5 detectors operating at design sensitivity. We can expect to observe many binary black hole, binary neutron star and (black hole, neutron star) mergers. This will lead to strong constraints on the population density of these objects, so providing guidance concerning formation channels. We can also expect good constraints on the deformability of neutron stars and thus of the equation of state. We will be able to test gravitation theory in the dynamical strong-field regime, leading to constraints on general relativity and alternatives. By the mid-2020s, pulsar timing can be expected to produce positive results, so providing information about supermassive black holes, and perhaps their formation channels. And by the mid-2030s, LISA will be operating and can be expected to provide a wealth of information for precision astrophysics and cosmology.

As we open a new window onto the Universe, we may observe something interesting and unexpected. Perhaps there will be guidance towards unifying gravitation and quantum mechanics, which has been unresolved for the last 85 years.

\section{Acknowledgments}

I thank the National Research Foundation, South Africa, for financial support; and Sabanci University, Turkey, for hospitality while writing this article. Some figures, as indicated in the captions, use material from the source cited. I thank the authors concerned for permitting this by making their publications open access under the creative commons license.

\section{References}

[1] LIGO And VIRgo collaboration, B. P. Abbott et. al., Prospects for Observing and Localizing Gravitational-Wave Transients with Advanced LIGO and Advanced Virgo, Living Rev. Relativ. 19 (2016) 1.

[2] LIGO AND VIRGo collaboration, B. P. Abbott et. al., Observation of Gravitational Waves from a Binary Black Hole Merger, Phys. Rev. Lett. 116 (2016) 061102, [1602.03837].

[3] A. Einstein, Naherungsweise Integration der Feldgleichungen der Gravitation, Sitzungsberichte der Königlich Preussischen Akademie der Wissenschaften (Berlin) 1916 (1916) 688-696.

[4] A. Einstein, Über Gravitationswellen, Sitzungsberichte der Königlich Preussischen Akademie der Wissenschaften (Berlin) 1918 (1918) 154-167.

[5] P. C. Peters and J. Mathews, Gravitational radiation from point masses in a Keplerian orbit, Phys. Rev. 131 (1963) 435-440.

[6] H. Bondi, M. G. J. van der Burg and A. W. K. Metzner, Gravitational waves in general relativity VII. Waves from axi-symmetric isolated systems, Proc. R. Soc. London A269 (1962) 21-52. 
[7] S. Chandrasekhar, The Mathematical Theory of Black Holes. Oxford University Press, Oxford, England, 1983.

[8] P. C. Peters, Gravitational radiation and the motion of two point masses, Phys. Rev. 136 (1964) B1224-B1232.

[9] LIGO AND VIRGo collaboration, B. P. Abbott et. al., GW151226: Observation of Gravitational Waves from a 22-Solar-Mass Binary Black Hole Coalescence, Phys. Rev. Lett. 116 (2016) 241103, [1606.04855].

[10] LIGO AND VIRGo collaboration, B. P. Abbott et. al., GW170104: Observation of a 50-Solar-Mass Binary Black Hole Coalescence at Redshift 0.2, Phys. Rev. Lett. 118 (2017) 221101, [1706.01812].

[11] LIGO AND VIRGo collaboration, B. P. Abbott et. al., GW170814: A Three-Detector Observation of Gravitational Waves from a Binary Black Hole Coalescence, Phys. Rev. Lett. 119 (2017) 141101, [1709.09660].

[12] LIGO AND VIRGO collaboration, B. P. Abbott et. al., GW170608: Observation of a 19 Solar-mass Binary Black Hole Coalescence, Astrophys. J. Lett. 851 (2017) L35, [1711 . 05578].

[13] V. Connaughton et. al., Fermi GBM Observations of LIGO Gravitational-wave Event GW150914, Astrophys. J. Lett. 826 (2016) L6, [1602.03920].

[14] LIGO AND VIRGO collaboration, B. P. Abbott et. al., GW170817: Observation of Gravitational Waves from a Binary Neutron Star Inspiral, Phys. Rev. Lett. 119 (2017) 161101, [1710. 05832].

[15] LIGO, VIRGO AND OTHERS collaboration, B. P. Abbott et. al., Multi-messenger Observations of a Binary Neutron Star Merger, Astrophys. J. Lett. 848 (2017) L12, [1710 . 05833].

[16] B. F. Schutz, Determining the hubble constant from gravitational wave observations, Nature 323 (1986) 310-311.

[17] LiGO, Virgo AND OTHERS collaboration, B. P. Abbott et. al., A gravitational-wave standard siren measurement of the Hubble constant, Nature 551 (2017) 85-88, [1710. 05835 ].

[18] Karsten Danzmann et al., "LISA Laser Interferometer Space Antenna." https://www.elisascience.org/files/publications/LISA_L3_20170120.pdf, 2016.

[19] Hellings, R.W.. and Downs, G.S., Upper limits on the isotropic gravitational radiation background from pulsar timing analysis, Astrophys. J. 265 (1983) 39.

[20] G. Hobbs and S. Dai, Gravitational wave research using pulsar timing arrays, National Science Review 4 (2017) 707-717, [1707.01615]. 